\title{
Adult Tarsal Scaphoiditis: Management
}

\author{
Rajesh Simon ${ }^{1}$, Ammanath N Sukesh $^{2}$, Dennis P Jose ${ }^{3}$
}

\begin{abstract}
Adult tarsal scaphoiditis or Mueller Weiss disease is a rare condition characterized by collapse of lateral half of the navicular bone. It is a spontaneous adult onset osteonecrosis of navicular bone with unclear etiology. Mueller Weiss disease is not sequelae to childhood onset Kohler's disease. Middle-aged women complain of midfoot and hindfoot pain. A detailed clinical and plain weight-bearing radiograph of foot is often diagnostic. The condition is graded using Maceira classification based on the deformity on lateral radiographs. Numerous surgical options are described for the management but no gold standard. We are presenting three patients, one with bilateral feet involvement, all in Maceira stage III deformity treated with double arthrodesis (talonavicular-cuneiform and calacaneocuboid) and autologous bone grafting achieving solid fusion and good functional outcome.
\end{abstract}

Keywords: Acetabular pedis, Adult tarsal scaphoditis, Avascular necrosis of navicular, Mueller Weiss disease.

Journal of Foot and Ankle Surgery (Asia Pacific) (2020): 10.5005/jp-journals-10040-1114

\section{INTRODUCTION}

A rare condition ${ }^{1}$ of unclear etiology characterized by collapse of lateral half of tarsal navicular bone or acetabulum pedis. Spontaneous adult onset osteonecrosis of navicular bone is a distinct entity and should not be confused with Kohler's disease, i.e., two names, same bone, but differs in patient age-group, clinical course and radiographic findings. It is to be clearly understood that Mueller Weiss disease is not a radiologic sequelae of Kohler's disease which occurs in childhood and is self-limiting. ${ }^{2}$

A disease that evolved through ages with different names and proposed etiology is delitescent, misdiagnosed, and improperly treated. The majority of patients are middle-aged females presenting with chronic pain in mid- and hindfoot. The etiology of the disease is still unclear, initially thought as a traumatic pathology then congenital, osteonecrosis, and now have numerous postulated etiopathogenesis. ${ }^{1,3}$ However, delayed ossification of tarsal navicular and abnormal force distribution remain the most widely accepted pathology.

\section{Materials and Methods}

We had three patients with four affected feet ( 1 with bilateral involvement). All patients were women with an average age of 50 years (range 43-56). Patients presented to our outpatient department with long-standing midfoot and hindfoot pain. There was no history of trauma nor any underlying pathology in our group of patients. Clinical evaluation revealed tenderness at navicular area more at the dorsolateral aspect. All patients were radiographed in full weight-bearing position and the deformed navicular bone was noted. The radiographic findings included loss of lateral half of navicular height (Fig. 1A), a comma-shaped navicular (Fig. 1A), dorsal protrusion with osteophytes (Fig. 1B), large open sinus tarsi (Fig. 1C), and fragmentation (Fig. 1D). Patients were staged according to Maceira classification based on talo-first metatarsal angle (MearyTomeno line) from lateral radiographic imaging (Fig. 2 and Table 1). All our patients had Maceira grade III radiographic changes. The condition was discussed with our patients and surgical treatment was advocated. We performed a double arthrodesis (talonavicularcuneiform and calcaneocuboid) (Fig. 3) through a medial and
${ }^{1-3}$ Department of Orthopaedics, VPS Lakeshore Global Lifecare, Kochi, Kerala, India

Corresponding Author: Rajesh Simon, Department of Orthopaedics, VPS Lakeshore Global Lifecare, Kochi, Kerala, India, Phone: +91 9447634466, e-mail: footandanklekochi@gmail.com

How to cite this article: Simon R, Sukesh AN, Jose DP. Adult Tarsal Scaphoiditis: Management. J Foot Ankle Surg (Asia Pacific) 2020;7(1):21-23.

Source of support: Nil

Conflict of interest: None

lateral approach (Fig. 4) in all our patients. All the arthrodesis was supplemented with bone graft from iliac crest. Medial arthrodesis was done with $4 \mathrm{~mm}$ headless compression screws and lateral using plate and screw. Patients were immobilized in a below knee slab in the immediate postoperative period.

\section{Results}

We advocated a postoperative protocol of immediate below knee slab for 2 weeks, suture removal at 2 weeks followed by slab conversion to a below knee cast, and then to boots. Patients were kept non-weight-bearing till 6 weeks of postoperative period. Full weight-bearing was advocated after 3 months. All patients were followed up at regular intervals of 1 month, 3, 6, and 12 months with clinical and radiographic evaluation. We have the longest follow-up of 3 years.

All the feet had solid union radiologically. We had no complications in our patients. The results were analyzed using American Orthopaedic Foot and Ankle Society (AOFAS) score, and we achieved an improved score from $39.5 / 100$ preoperatively to $88 / 100$ postoperatively (Table 2 ).

\section{Discussion}

Mueller Weiss disease or Brailsford disease ${ }^{4}$ is a rare pathology in adults affecting tarsal navicular bone. Adult onset spontaneous osteonecrosis of navicular is common in females and usually

() The Author(s). 2020 Open Access This article is distributed under the terms of the Creative Commons Attribution 4.0 International License (https://creativecommons. org/licenses/by-nc/4.0/), which permits unrestricted use, distribution, and non-commercial reproduction in any medium, provided you give appropriate credit to the original author(s) and the source, provide a link to the Creative Commons license, and indicate if changes were made. The Creative Commons Public Domain Dedication waiver (http://creativecommons.org/publicdomain/zero/1.0/) applies to the data made available in this article, unless otherwise stated. 

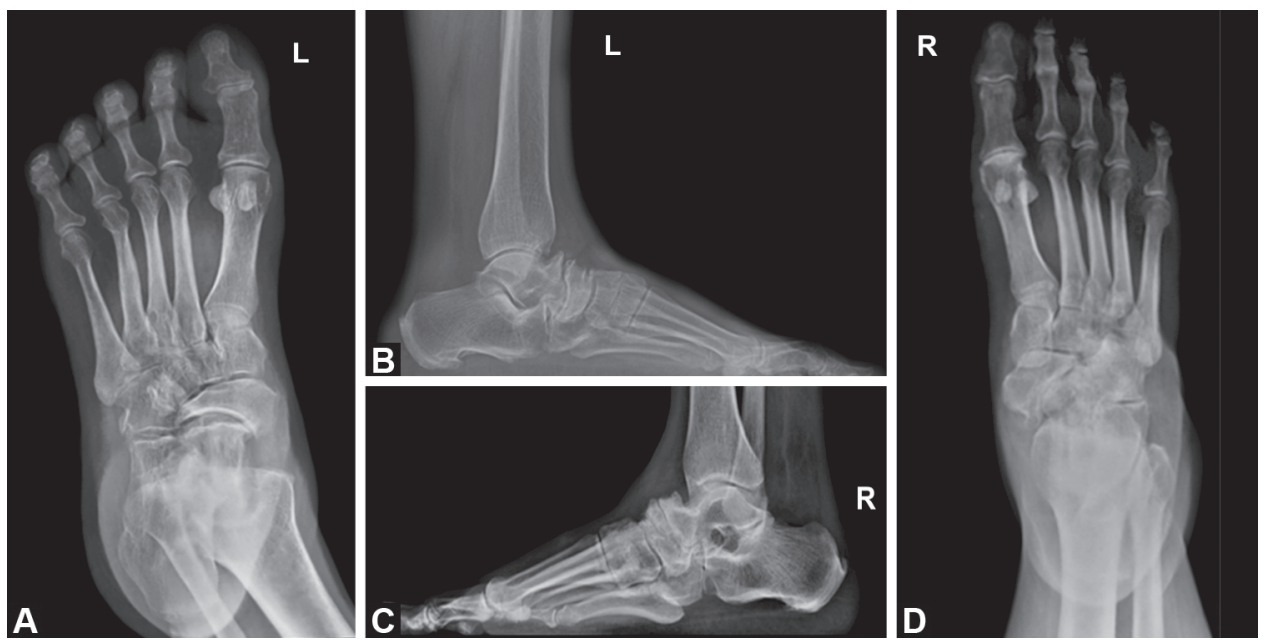

Figs 1 A to D: Radiographic features of Mueller Weiss disease: (A) Comma-shaped navicular; (B) Dorsal protrusion with osteophytes; (C) Large open sinus tarsi; (D) Fragmentation of navicular bone

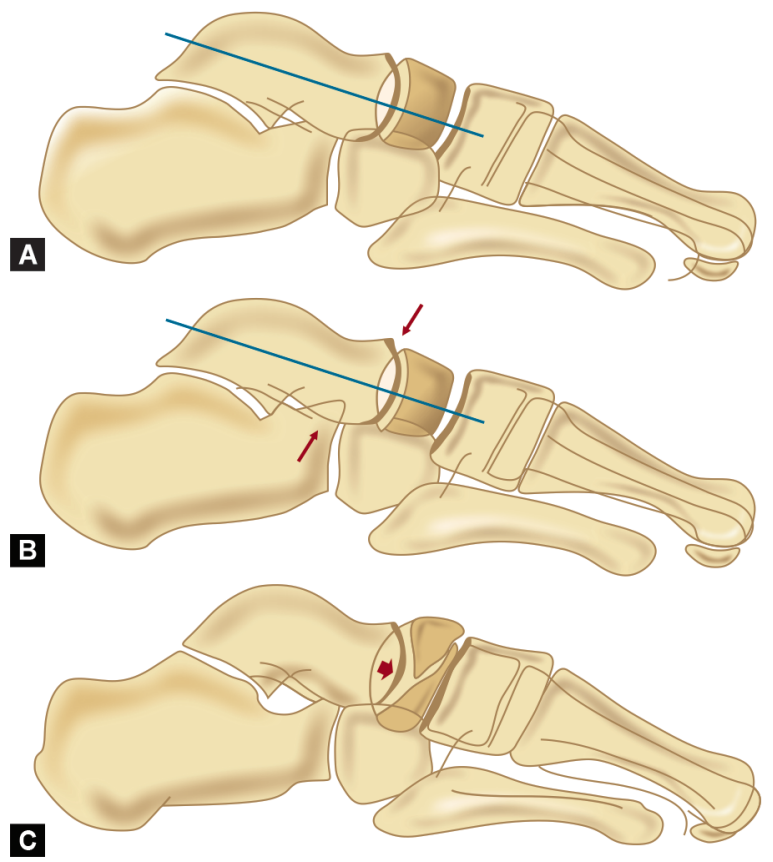

Table 1: Maceira classification based on plain lateral radiographic imaging

\begin{tabular}{|c|c|c|c|}
\hline Stage & Incidence (\%) & Radiograph & Meary-Tomeno line \\
\hline I & 19 & Normal & Straight \\
\hline II & 9 & $\begin{array}{l}\text { Dorsally subluxated } \\
\text { talus head }\end{array}$ & Dorsal angulation \\
\hline III & 46 & $\begin{array}{l}\text { Compression } \\
\text { lowering of } \\
\text { longitudinal arch }\end{array}$ & Neutral/straight \\
\hline IV & 19 & $\begin{array}{l}\text { Fragmentation flat } \\
\text { longitudinal arch }\end{array}$ & Plantar \\
\hline V & 7 & $\begin{array}{l}\text { Extruded navicular } \\
\text { talocuneiform joint }\end{array}$ & Plantar \\
\hline
\end{tabular}

radiographic weight-bearing imaging are often diagnostic. ${ }^{6}$ The disease is classified into five stages by Maceira, ${ }^{1}$ on the basis of deformity on plain lateral radiographs and assessing the MearyTomeno line.

The rarity of this condition limits the standard of treatment. A forgotten cause of midfoot pain with unclear etiology and no gold standard treatment. As in any foot and ankle condition, the treatment starts with a trial of conservative measures for early stages of the disease but nothing stops the progression of the disease. A failed conservative treatment/severe midfoot deformity lead to surgical management. ${ }^{7}$ A handful of surgical trial and error methods like percutaneous core decompression, isolated talonavicular, talonavicular-cuneiform, ${ }^{8}$ triple arthrodesis ${ }^{9,10}$ have been advocated but no gold standards. The common goal of achieving pain relief and restoration of longitudinal arches is best provided by the talonavicular-cuneiform arthrodesis with bone grafting. ${ }^{11}$

\section{Conclusion}

Mueller Weiss syndrome is an unusual cause of midfoot pain, a diagnostic challenge. It is an uncommon disease with controversial etiology and without an established standard of treatment. We advocate double arthrodesis (addressing naviculocuneiform) with autologous bone grafting a treatment solution for grade III Mueller Weiss disease as it has good functional outcome and high fusion rate. 

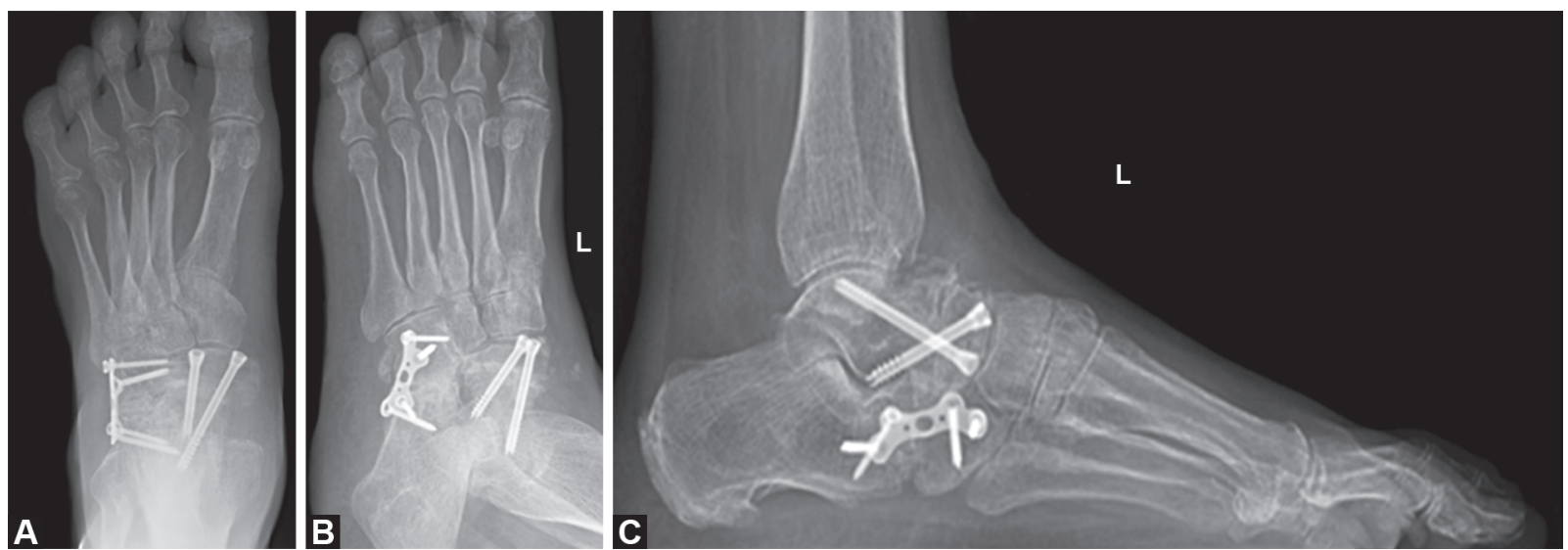

Figs $3 \mathrm{~A}$ to $\mathrm{C}$ : Double arthrodesis anteroposterior, oblique, and lateral postoperative radiographs
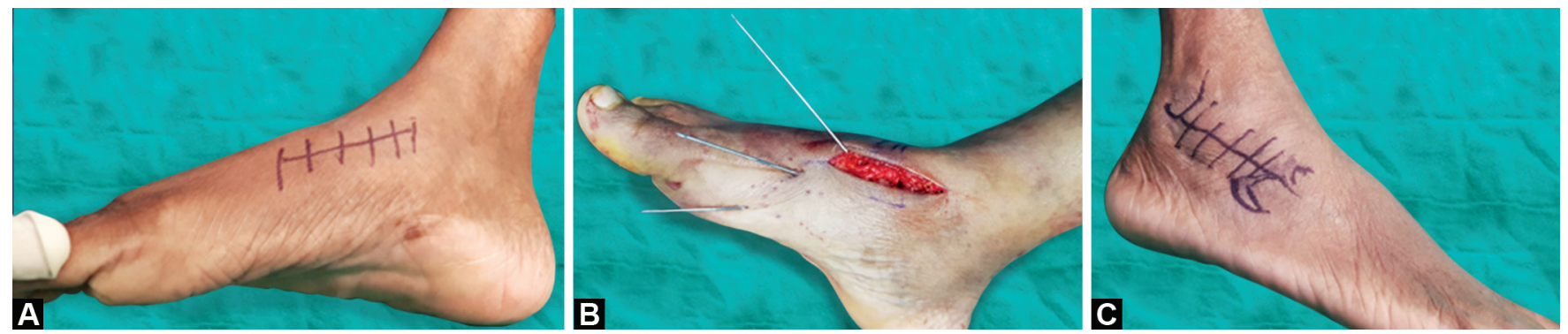

Figs 4A to C: Intraoperative picture with medial and lateral incisions

Table 2: Patient details

\begin{tabular}{lllllll}
\hline & & & & & AOFAS & AOFAS \\
& Age & Sex & Foot & Stage Maceira & preoperative score & postoperative score \\
\hline Patient 1 & 43 & Female & Left & III & 40 & 86 \\
Patient 1 & 43 & Female & Right & III & 38 & 90 \\
Patient 2 & 56 & Female & Left & III & 44 & 92 \\
Patient 3 & 55 & Female & Left & III & 36 & 86 \\
\hline
\end{tabular}

\section{LIMITATIONS}

Our study consisted of a small sample size. Fusion of bone was assessed using plain radiographs instead of computed tomography scans. We are looking forward to collect data from other centers to have enough sample size, so that we can have a better understanding and analysis.

\section{References}

1. Maceira E, Rochera R. Muller-Weiss disease: clinical and biomechanical features. Foot Ankle Clin 2004;9(1):105-125. DOI: 10.1016/S10837515(03)00153-0.

2. Ippolito E, Ricciardi Pollini PT, Falez F. Kohler's disease of the tarsal navicular: long term follow-up of 12 cases. J Pediatr Orthop 1984;4(4):416-417. DOI: 10.1097/01241398-198408000-00004.

3. Lafontaine $\mathrm{M}, \mathrm{Charlier} \mathrm{PH}, \mathrm{Hardy} \mathrm{D}$, et al. Hypermobility of the 1st ray associated with osteonecrosis-fracture of the tarsal navicular bone. Acta Orthop Belg 1989;55(4):565-571.

4. Brailsford F. Osteochondritis of the adult tarsal navicular. J Bone Joint Surg 1939;21-A:III-120.
5. Tosun B, AI F, Tosun A. Spontaneous osteonecrosis of the tarsal navicular in an adult: Mueller-Weiss syndrome. J Foot Ankle Surg 2001;50(2):221-224. DOI: 10.1053/j.jfas.2010.10.019.

6. Samim M, Moukaddam HA, Smitaman E. Imaging of MuellerWeiss syndrome: a review of clinical presentations and imaging spectrum. AJR Am J Roentgenol 2016;207(2):W8-W18. DOI: 10.2214/ AJR.15.15843.

7. Doyle T, Napier RJ, Wong-Chung J. Recognition and management of Muller-Weiss disease. Foot Ankle Int 2012;33(4):275-281. DOI: 10.3113/ FAl.2012.0275.

8. Yu G, Zhao Y, Zhou J, et al. Fusion of talona-vicular and naviculocuneiform joints for the treatment of Muller-Weiss disease. J Foot Ankle Surg 2012;51(4):415-419. DOI: 10.1053/j.jfas.2012.04.004.

9. Lui TH. Arthroscopic triple arthrodesis in patients with muller Weiss disease. Foot Ankle Surg 2009;15(3):119-122V. DOI: 10.1016/ j.fas.2008.08.010.

10. Cao H-H, Tang K-L, Xu J-Z. Peri-navicular ar- throdesis for the stage III Muller-Weiss disease. Foot Ankle Int 2012;33(6):475-478. DOI: 10.3113/ FAl.2012.0475.

11. Zhang $\mathrm{H}$, Li J, Qiao Y. Open triple fusion versus TNC arthrodesis in the treatment of Mueller-Weiss disease. J Orthop Surg 2017;12(1):13. DOI: 10.1186/s13018-017-0513-3. 